\title{
LAPLACE TRANSFORM GENERATION THEOREMS AND LOCAL CAUCHY PROBLEMS
}

\author{
CLAUS MÜLLER
}

Received 30 April 2001

We give new criterions to decide if some vector-valued function is a local Laplace transform and apply this to the theory of local Cauchy problems. This leads to an improvement of known results and new Hille-Yosida-type theorems for local convoluted semigroups.

\section{Introduction}

Let $X$ be a Banach space, $x \in X, T>0, h \in L_{1}([0, T], \mathbb{K})$, and $f(t):=\int_{0}^{t} h(s) d s$ the antiderivative of $h$.

Assume $A: D(A) \rightarrow X$ is some closed (linear) operator in $X$.

Consider the abstract Cauchy problem

(ACP) $A w(t)=w^{\prime}(t)$ if $0 \leq t \leq T, w(0)=x$, and $w \in C^{1}([0, T], X)$

and the $f$-regularized problem

$\left(\mathrm{ACP}_{f}\right) A u(t)+f(t) x=u^{\prime}(t)$ if $0 \leq t \leq T, u(0)=0$, and $u \in C^{1}([0, T], X)$.

If $w$ solves (ACP), then the convolution $u:=w * f$ solves $\left(\mathrm{ACP}_{f}\right)$, since

$$
u^{\prime}=w^{\prime} * f+f(\cdot) x=w * h \quad \text { on }[0, T] .
$$

Any function $u$ that solves $\left(\mathrm{ACP}_{f}\right)$ is called $h$-regularized solution of (ACP).

Now assume that $0 \in \operatorname{supp} h$, which means that $h$ does not vanish on any interval $[0, \varepsilon)$, and which is equivalent to $0 \in \operatorname{supp} f$.

By the theorem of Titchmarsh-Foiaş (see [3]) the convolution operator

$$
\begin{gathered}
S_{f}: C([0, T], X) \longrightarrow C_{0}([0, T], X)=\{g \in C([0, T), X) \mid g(0)=0\}, \\
S_{f} g=g * f,
\end{gathered}
$$

can be extended to an isometric isomorphism

$$
\widetilde{S}_{f}: C^{[f]}([0, T], X) \longrightarrow C_{0}([0, T], X),
$$

Copyright (C) 2004 Hindawi Publishing Corporation

Abstract and Applied Analysis 2004:1 (2004) 69-90

2000 Mathematics Subject Classification: 47D03, 47D06, 44A10

URL: http://dx.doi.org/10.1155/S1085337504309073 
where the space $C^{[f]}([0, T], X)$ of generalized functions is the completion of $C([0, T], X)$ with norm $\|g\|:=\left\|S_{f} g\right\|_{\infty}$. On this construction see also [9]. Thus, if $w$ solves (ACP), then $S_{f} w$ solves $\left(\mathrm{ACP}_{f}\right)$.

This gives a reason to extend the notion of solutions of (ACP): a generalized function $w \in C^{[f]}([0, T], X)$ is called $h$-generalized solution of

$\left(\mathrm{ACP}_{*}\right) A w(t)=w^{\prime}(t)$ if $0 \leq t \leq T, w(0)=x$

if $u=\tilde{S}_{f} w$ solves $\left(\mathrm{ACP}_{f}\right)$, that is, if $\tilde{S}_{f} w$ is an $h$-regularized solution of (ACP).

The notation of generalized solutions was introduced by Cioranescu and Lumer $[5,6]$.

If $w$ is an $h$-generalized solution of $\left(\mathrm{ACP}_{*}\right)$, there is a sequence $\left(v_{n}\right)_{n} \subset C([0, T]$, $\bar{D}(A))$ with $\lim _{v} v_{n}=u^{\prime}$ in $C([0, T], X)$ and with $\lim _{n} v_{n}^{[1]}=u$ in $C([0, T], \bar{D}(A))$ (where $\bar{D}(A)$ denotes the Banach space $D(A)$ with the graph norm).

Thus, $\lim _{n} v_{n}=u^{\prime}$, and $\lim _{n}\left(A v_{n}^{[1]}+f(\cdot) x-v_{n}\right)=0$ in $C([0, T], X)$.

If $\left(v_{n}\right)_{n} \subset C([0, T], X)$ converges uniformly and satisfies $v_{n}^{[1]}(t) \in D(A)$ for all $t$ and $\lim _{n}\left(A v_{n}^{[1]}+f(\cdot) x-v_{n}\right)=0$ uniformly, then $\left(v_{n}\right)_{n}$ is called $h$-approximate solution of (ACP). Thus to every $h$-generalized solution there is an $h$-approximate solution.

On the other hand, if $\left(v_{n}\right)_{n}$ is an $h$-approximate solution of (ACP), then $w_{n}=\widetilde{S}_{h}^{-1}\left(v_{n}-\right.$ $\left.v_{n}(0)\right)$ converges in $C^{[h]}([0, T], X)$ to some $w$, and $u=\widetilde{S}_{f} w=\lim _{n} v_{n}^{[1]}$ solves $\left(\mathrm{ACP}_{f}\right)$. On approximate solutions see $[1,2]$. Consequently, the notations of generalized solution of $\left(\mathrm{ACP}_{*}\right)$, of approximate solutions of $(\mathrm{ACP})$, and (classical) solutions of $\left(\mathrm{ACP}_{f}\right)$ are equivalent. That is the reason why it is interesting to study problems of type $\left(\mathrm{ACP}_{f}\right)$.

We first clarify the notations.

If $X$ is some Banach space, $g \in L_{1}^{\text {loc }}([0, \infty), X)$, and $\alpha, \tau>0$, we let

$$
g^{(-\alpha)}(t):=g^{[\alpha]}(t):=\frac{1}{\Gamma(\alpha)} \int_{0}^{t} s^{\alpha-1} g(t-s) d s
$$

be the $\alpha$ th integral of $g$. If there is some $h \in C([0, \tau], X)$ with $g=h^{[\alpha]}$, then let $g^{(\alpha)}=h$ be the $\alpha$ th derivative of $g$.

The finite Laplace transform of $g$ on $[0, \tau]$ is given by

$$
\widehat{g}_{\tau}(\lambda)=g_{\tau}^{\wedge}(\lambda):=\int_{0}^{\tau} e^{-\lambda t} g(t) d t
$$

If $\alpha>0$ and $g$ is exponentially bounded for large arguments, then $g^{[\alpha]}$ is exponentially bounded for large arguments, and

$$
\lambda^{\alpha} \int_{0}^{\infty} e^{-\lambda t} g^{[\alpha]}(t) d t=\int_{0}^{\infty} e^{-\lambda t} g(t) d t=: \hat{g}(\lambda)=g^{\wedge}(\lambda)
$$

for all large Re $\lambda$, which is the Laplace transform of $g$.

In fact we have, if $\tau>0$,

$$
\lambda^{\alpha} \int_{0}^{\tau} e^{-\lambda t} g^{[\alpha]}(t) d t \sim_{\tau} \int_{0}^{\tau} e^{-\lambda t} g(t) d t
$$


where

$$
\begin{aligned}
f(\lambda) \sim_{\tau} h(\lambda) & \Longleftrightarrow \limsup _{\lambda \rightarrow \infty} \frac{\ln |f(\lambda)-h(\lambda)|}{\lambda} \leq-\tau \\
& \Longleftrightarrow \lim _{\lambda \rightarrow \infty} e^{-\delta \lambda} e^{\lambda \tau}|f(\lambda)-h(\lambda)|=0 \quad \forall \delta>0 .
\end{aligned}
$$

This follows from

$$
\begin{aligned}
\lambda^{\alpha} \int_{0}^{\tau} e^{-\lambda t} g^{[\alpha]}(t) d t & =\frac{\lambda^{\alpha}}{\Gamma(\alpha)}\left(p_{\alpha-1} * g\right)_{\tau}^{\wedge}(\lambda), \quad \text { where } p_{\beta}(s)=s^{\beta} \\
& \sim_{\tau} \frac{\lambda^{\alpha}}{\Gamma(\alpha)}\left(p_{\alpha-1}\right)^{\wedge}(\lambda) \cdot g_{\tau}^{\wedge}(\lambda)=g_{\tau}^{\wedge}(\lambda) .
\end{aligned}
$$

In trying to get a nice theory how to solve abstract Cauchy problems

$$
\begin{gathered}
A u_{x}(t)+f(t) x=u_{x}^{\prime}(t) \quad \text { if } 0 \leq t \leq \tau, \\
u_{x}(0)=0, \\
u_{x} \in C^{1}([0, \tau], X),
\end{gathered}
$$

with continuous inhomogeneity $f$, it seems to be natural first to consider inhomogeneities of type $t^{\beta}$, where $\beta \in \mathbb{N}$ or, more generally, $\beta \geq 0$. The reason for this is that if the problem is well posed on $[0, \tau]$ with this inhomogeneity, that is, $A$ generates a $\beta$ integrated semigroup on $[0, \tau]$, then it is well posed with inhomogeneities $h^{[\beta+1]}$, where $h \in L_{1}([0, \tau], \mathbb{K})$, that is, $A$ generates a local $h^{[\beta+1]}$-convoluted semigroup on $[0, \tau]$. This is shown in Section 2.

Section 3 starts with a generalization of the complex representation theorem, see $[4,12]$. This will lead to an improvement of [7, Theorem II].

In Section 4, we generalize a representation theorem of Prüss [12] and develop a new Hille-Yosida-type theorem for integrated semigroups.

\section{Integrated semigroups}

In this section, we show that it is worthwhile to study integrated semigroups, that is, abstract Cauchy problems $\left(\mathrm{ACP}_{f}\right)$ with inhomogeneities $f(t)=t^{\beta}$.

If $\tau>0$, we say that a subspace $Y \subset L_{1}([0, \tau], \mathbb{K})$ satiesfies property (A) if $Y$ is closed and there is some strictly decreasing null sequence $\left(\varepsilon_{n}\right)_{n}$ with $\mathbf{1}_{\left[0, \varepsilon_{n}\right]} \in Y$ for all $n$.

The smallest possible $Y$ with property (A) is

$$
Y=\left\{\sum_{n=1}^{\infty} \alpha_{n} \mathbf{1}_{\left(\varepsilon_{n+1}, \varepsilon_{n}\right)} \mid\left(\alpha_{n}\right)_{n} \in \ell_{\infty}\right\},
$$

where $\mathbf{1}_{(a, b)}$ is the characteristic function on $(a, b)$.

Theorem 2.1. Let $X$ be a real or complex Banach space, $A: D(A) \rightarrow X$ a closed linear operator, $\tau>0$, and $Y \subset L_{1}([0, \tau], \mathbb{K})$ some subspace with property $(A)$. 
72 Laplace transform and local Cauchy problems

Let $\beta \geq 0$, and assume A generates a local $h^{[\beta+1]}$-convoluted semigroup on $[0, \tau]$ for all $h \in Y$.

Then $A_{\overline{D(A)}}$ generates a $\beta$-times-integrated semigroup on $[0, \tau]$.

Proof. If $x \in X$ and $h \in Y$, let $u_{x, h}$ denote the solution of the abstract Cauchy problem

$$
\begin{gathered}
A u_{x, h}(t)+h^{[\beta+1]}(t) x=u_{x, h}^{\prime}(t) \quad \text { if } 0 \leq t \leq \tau, \\
u_{x, h}(0)=0 \\
u_{x, h} \in C^{1}([0, \tau], X) .
\end{gathered}
$$

Using the closedness of $A$ and the uniqueness property of the abstract Cauchy problem, it is easy to see that the linear operator

$$
\begin{gathered}
R_{h}: X \longrightarrow C([0, \tau], X), \\
R_{h} x=u_{x, h}^{\prime},
\end{gathered}
$$

has closed graph for all $h \in Y$.

Moreover, for all $x \in X$, the linear operator

$$
\begin{gathered}
S_{x}: Y \longrightarrow C([0, \tau], X), \\
S_{x} h=u_{x, h}^{\prime},
\end{gathered}
$$

has closed graph.

From the uniform boundedness principle, it follows that

$$
\sup _{\|x\| \leq 1}\left\|S_{x}\right\|<\infty
$$

consequently

$$
\sup _{\|x\| \leq 1} \sup _{\|h\|_{Y} \leq 1}\left\|u_{x, h}^{\prime}\right\|_{\infty}<\infty
$$

Now, let $h_{n}:=\left(1 / \varepsilon_{n}\right) \cdot \mathbf{1}_{\left[0, \varepsilon_{n}\right]}$.

Then

$$
\Gamma(\beta+1) \cdot h_{n}^{[\beta+1]}(t)= \begin{cases}\frac{1}{\varepsilon_{n}} \frac{t^{\beta+1}}{\beta+1} & \text { if } 0 \leq t \leq \varepsilon_{n}, \\ \frac{1}{\beta+1} \frac{t^{\beta+1}-\left(t-\varepsilon_{n}\right)^{\beta+1}}{\varepsilon_{n}} & \text { if } \varepsilon_{n} \leq t \leq \tau,\end{cases}
$$

thus $\lim _{n \rightarrow \infty} h_{n}^{[\beta+1]}(t)=t^{\beta} / \Gamma(\beta+1)$ if $0<t \leq \tau$.

Moreover, $\sup _{n}\left\|h_{n}^{[\beta+1]}\right\|_{\infty}<\infty$. From the dominated convergence theorem, it follows that

$$
\int_{0}^{\tau}\left|h_{n}^{[\beta+1]}(t)-\frac{t^{\beta}}{\Gamma(\beta+1)}\right| d t \stackrel{n}{\longrightarrow} 0
$$


Next, we show that

(a) $\lim _{\lambda, n \rightarrow \infty} e^{\lambda \tau}\left|\left(h_{n}^{[\beta+1]}\right)_{\tau}^{\wedge}(\lambda)\right|=\infty$,

(b) $\liminf _{\lambda \rightarrow \infty}\left(\ln \left|\left(t^{\beta} / \Gamma(\beta+1)\right)_{\tau}^{\wedge}(\lambda)\right| / \lambda\right) \geq 0$.

We prove (a). We have

$$
\sup _{\lambda, n} e^{\lambda \tau}\left|\left(h_{n}^{[\beta+1]}\right)^{\wedge}(\lambda)-\left(h_{n}^{[\beta+1]}\right)_{\tau}^{\wedge}(\lambda)\right|<\infty
$$

since

$$
\left|\int_{\tau}^{\infty} e^{-\lambda t} h_{n}^{[\beta+1]}(t) d t\right| \leq \sup _{n}\left\|h_{n}^{[\beta+1]}\right\|_{\infty} \cdot \int_{\tau}^{\infty} e^{-\lambda t} d t
$$

Moreover,

$$
e^{\lambda \tau}\left|\left(h_{n}^{[\beta+1]}\right)_{\tau}^{\wedge}(\lambda)\right| \geq e^{\lambda \tau}\left|\left(h_{n}^{[\beta+1]}\right)^{\wedge}(\lambda)\right|-e^{\lambda \tau}\left|\left(h_{n}^{[\beta+1]}\right)^{\wedge}(\lambda)-\left(h_{n}^{[\beta+1]}\right)_{\tau}^{\wedge}(\lambda)\right|
$$

Now, (a) follows from

$$
\left(h_{n}^{[\beta+1]}\right)^{\wedge}(\lambda)=\frac{\widehat{h_{n}}(\lambda)}{\lambda^{\beta+1}}=\frac{1}{\lambda^{\beta+2}} \frac{1-e^{-\lambda \varepsilon_{n}}}{\varepsilon_{n}} \geq \frac{e^{-\lambda \varepsilon_{n}}}{\lambda^{\beta+1}} \geq \frac{e^{-\lambda \tau / 2}}{\lambda^{\beta+1}} \quad \text { for all large } \lambda, n .
$$

We prove (b). This follows from [10, Lemma 3.3.5] or [8, Lemma 2.6].

Now we can apply [11, Corollary 13, Theorem 14], which yields the desired results.

Corollary 2.2. Let $X$ be a real or complex Banach space, $A: D(A) \rightarrow X$ a densely defined closed linear operator, $\tau>0$, and $\beta \geq 0$.

Then the following assertions are equivalent:

(1) A generates a local $\beta$-times-integrated semigroup on $[0, \tau]$;

(2) A generates a local $h^{[\beta+1]}$-convoluted semigroup on $[0, \tau]$ whenever $h \in L_{1}([0, \tau], \mathbb{K})$;

(3) for one (for all) subspace(s) $Y \subset L_{1}([0, \tau], \mathbb{K})$ with property $(A)$, the operator A generates a local $h^{[\beta+1]}$-convoluted semigroup on $[0, \tau]$ whenever $h \in Y$.

Proof. The proof of (2) follows from (1) by a simple convolution argument even if $D(A)$ is not dense.

\section{The complex representation theorem and its application}

In the following, we generalize the complex representation theorem to the local case. The global version can be found in [4] or [12, Proposition 0.2].

Theorem 3.1 (complex representation theorem). Let $X$ be a complex Banach space, $c, a$, $\omega_{1}>0, F(t):=c\left(e^{a t}-1\right)$, and $M:=\left\{z \in \mathbb{C}\left|\operatorname{Re} z>\omega_{1},\right| \operatorname{Im} z \mid<F\left(\operatorname{Re} z-\omega_{1}\right)\right\}$.

Furthermore, let $q: M \rightarrow X$ be a holomorphic function satisfying

$$
\sup _{\lambda \in M}\|\lambda q(\lambda)\|<\infty
$$


74 Laplace transform and local Cauchy problems

Then for every $b>0$ there is some function $g_{b} \in C([0, a b), X)$ such that $t \mapsto((a b-t) /$ $\left.t^{b}\right) g_{b}(t)$ is bounded on $(0, a b)$ and with

$$
q(\lambda) \sim_{\xi} \lambda^{b} \cdot\left(g_{b}\right)_{\xi}^{\wedge}(\lambda) \quad \forall \xi \in(0, a b)
$$

Remark 3.2. If $0<b^{\prime}<b$, then $g_{b}=g_{b^{\prime}}^{\left[b-b^{\prime}\right]}$ on $\left[0, a b^{\prime}\right)$.

Proof. Let $\omega>\omega_{1}, b>0$, and $\Gamma:=\{z \in \mathbb{C}|\operatorname{Re} z \geq \omega,| \operatorname{Im} z \mid \leq F(\operatorname{Re} z-\omega)\}$. Then $\Gamma=\bar{\Gamma} \subset$ $M^{0}=M$.

First, we note that

$$
C_{1}^{2}:=\sup _{r>0} \frac{1+\left(F^{\prime}(r)\right)^{2}}{\left[(\omega+r)^{2}+F(r)^{2}\right]^{b+1}} \cdot e^{2 a b r}<\infty .
$$

Let $C_{2}:=\sup _{\lambda \in \Gamma}\|\lambda q(\lambda)\|$. We parameterize $\partial \Gamma$ by the following two functions:

$$
\begin{array}{ll}
\gamma^{+}:[0, \infty) \longrightarrow \mathbb{C}, & \gamma^{+}(t)=\omega+t+i F(t) \\
\gamma^{-}:[0, \infty) \longrightarrow \mathbb{C}, & \gamma^{-}(t)=\omega+t-i F(t)
\end{array}
$$

Let $\gamma_{n}^{+}:=\left.\gamma^{+}\right|_{[0, n]}$ and $\gamma_{n}^{-}:=\left.\gamma^{-}\right|_{[0, n]}$. Then the sequence

$$
\begin{gathered}
g_{n}^{+}:[0, \infty) \longrightarrow X, \\
g_{n}^{+}(t)=\frac{1}{2 \pi i} \int_{\gamma_{n}^{+}} e^{\lambda t} \frac{q(\lambda)}{\lambda^{b}} d \lambda,
\end{gathered}
$$

of continuous functions converges uniformly on $\left[0, q^{\prime} b\right]$ whenever $q^{\prime} \in(0, a)$, since if $t \in\left[0, q^{\prime} b\right]$ and $n>m$, we obtain

$$
\begin{aligned}
2 \pi\left\|g_{n}^{+}(t)-g_{m}^{+}(t)\right\| & \leq\left\|\int_{m}^{n} e^{t \gamma^{+}(r)} \frac{q\left(\gamma^{+}(r)\right)}{\gamma^{+}(r)^{b}} \cdot \frac{d}{d r} \gamma^{+}(r) d r\right\| \\
& \leq C_{2} \int_{m}^{n} e^{t(\omega+r)} \frac{\sqrt{1+F^{\prime}(r)^{2}}}{\left[(\omega+r)^{2}+F(r)^{2}\right]^{(b+1) / 2}} d r \\
& \leq C_{1} C_{2} e^{t \omega} \int_{m}^{n} e^{t r} e^{-a b r} d r \\
& \leq C_{1} C_{2} e^{t \omega} \int_{m}^{n} e^{r b\left(q^{\prime}-a\right)} d r .
\end{aligned}
$$

Thus the function

$$
\begin{gathered}
g^{+}:[0, a b) \longrightarrow X, \\
g^{+}(t)=\frac{1}{2 \pi i} \int_{\gamma^{+}} e^{\lambda t} \frac{q(\lambda)}{\lambda^{b}} d \lambda,
\end{gathered}
$$


is continuous. In the same way we see that the function

$$
\begin{aligned}
& g^{-}:[0, a b) \longrightarrow X, \\
& g^{-}(t)=\frac{1}{2 \pi i} \int_{-\gamma^{-}} e^{\lambda t} \frac{q(\lambda)}{\lambda^{b}} d \lambda=-\frac{1}{2 \pi i} \int_{\gamma^{-}} e^{\lambda t} \frac{q(\lambda)}{\lambda^{b}} d \lambda,
\end{aligned}
$$

is continuous.

Let $g(t):=g^{+}(t)+g^{-}(t)$ if $t \in[0, a b)$. We show that $t \mapsto\left((a b-t) / t^{b}\right) g(t)$ is bounded on $(0, a b)$. To this end, let $R>0$ and consider the three paths

$$
\begin{aligned}
\alpha:[R, \infty) & \longrightarrow \mathbb{C}, \quad \alpha(r)=\omega+r-i F(r), \\
\beta:[R, \infty) & \longrightarrow \mathbb{C}, \quad \alpha(r)=\omega+r+i F(r), \\
\gamma:[-F(\omega+R), F(\omega+R)] & \longrightarrow \mathbb{C}, \quad \gamma(r)=\omega+R+i r .
\end{aligned}
$$

Then, if $0 \leq t<a b$,

$$
g(t)=\frac{1}{2 \pi i} \int_{\beta+\gamma-\alpha} e^{\lambda t} \frac{q(\lambda)}{\lambda^{b}} d \lambda .
$$

We have

$$
\begin{gathered}
\left\|\int_{\beta} e^{\lambda t} \frac{q(\lambda)}{\lambda^{b}} d \lambda\right\| \leq C_{1} C_{2} e^{\omega t} \int_{R}^{\infty} e^{(t-a b) r} d r=C_{1} C_{2} e^{\omega t} \frac{e^{(t-a b) R}}{a b-t} \\
\left\|\int_{-\alpha} e^{\lambda t} \frac{q(\lambda)}{\lambda^{b}} d \lambda\right\| \leq C_{1} C_{2} e^{\omega t} \frac{e^{(t-a b) R}}{a b-t}
\end{gathered}
$$

Finally,

$$
\begin{aligned}
\left\|\int_{\gamma} e^{\lambda t} \frac{q(\lambda)}{\lambda^{b}} d \lambda\right\| & \leq \int_{-\infty}^{\infty} e^{t(\omega+R)} C_{2} \frac{d r}{\left[(\omega+R)^{2}+r^{2}\right]^{(b+1) / 2}} \\
& =\frac{C_{2} e^{t(\omega+R)}}{(\omega+R)^{b}} \int_{-\infty}^{\infty} \frac{d s}{\left(1+s^{2}\right)^{(b+1) / 2}} .
\end{aligned}
$$

If we let $R:=1 / t$, we obtain the desired result.

Next, we show that, if $\lambda \in \Gamma$,

$$
\frac{q(\lambda)}{\lambda^{b}}=\frac{1}{2 \pi i} \lim _{n \rightarrow \infty} \int_{\gamma_{n}^{+}-\gamma_{n}^{-}} \frac{\left(1 / \mu^{b}\right) q(\mu)}{\lambda-\mu} d \mu .
$$

To this end, consider a path $\beta_{R}$ in $\mathbb{C}$ consisting of a part of a circle with center $\omega \in \mathbb{C}$ and radius $R$ which connects a point on $\gamma^{+}$with a point on $\gamma^{-}$. Its parameterization is given by

$$
\beta_{R}:[-\psi, \psi] \longrightarrow \mathbb{C}, \quad \beta_{R}(\varphi)=\omega+\operatorname{Re}^{i \varphi},
$$

with some $\psi \in(0, \pi / 2)$ depending on $R$. 
76 Laplace transform and local Cauchy problems

By Cauchy's formula we have to show that

$$
\lim _{R \rightarrow \infty} \int_{\beta_{R}} \frac{\left(1 / \mu^{b}\right) q(\mu)}{\mu-\lambda} d \mu=0 .
$$

But, if $R$ is large enough,

$$
\begin{aligned}
\left\|\int_{\beta_{R}} \frac{\left(1 / \mu^{b}\right) q(\mu)}{\mu-\lambda} d \mu\right\| & \leq \int_{-\pi / 2}^{\pi / 2} \frac{\left\|q\left(\omega+\operatorname{Re}^{i \varphi}\right)\right\|}{\left|\omega+\operatorname{Re}^{i \varphi}\right|^{b} \cdot\left|\lambda-\left(\omega+\operatorname{Re}^{i \varphi}\right)\right|} R d \varphi \\
& \leq C_{2} \int_{-\pi / 2}^{\pi / 2} \frac{R}{\left|\omega+\operatorname{Re}^{i \varphi}\right|^{b+1}} d \varphi \stackrel{R \rightarrow \infty}{\longrightarrow} 0 .
\end{aligned}
$$

Consequently, if $\xi \in(0, a b)$ and $\lambda \in \Gamma$,

$$
\begin{aligned}
\int_{0}^{\xi} e^{-\lambda t} g(t) d t= & \frac{1}{2 \pi i} \int_{0}^{\xi} e^{-\lambda t} \lim _{n \rightarrow \infty} \int_{\gamma_{n}^{+}-\gamma_{n}^{-}} e^{\mu t} \frac{q(\mu)}{\mu^{b}} d \mu d t \\
= & \frac{1}{2 \pi i} \lim _{n} \int_{\gamma_{n}^{+}-\gamma_{n}^{-}} \int_{0}^{\xi} e^{(\mu-\lambda) t} d t \frac{q(\mu)}{\mu^{b}} d \mu \\
= & \frac{1}{2 \pi i} \lim _{n} \int_{\gamma_{n}^{+}-\gamma_{n}^{-}} \frac{e^{(\mu-\lambda) \xi}}{\mu-\lambda} \frac{q(\mu)}{\mu^{b}} d \mu \\
& +\frac{1}{2 \pi i} \lim _{n} \int_{\gamma_{n}^{+}-\gamma_{n}^{-}} \frac{\left(1 / \mu^{b}\right) q(\mu)}{\lambda-\mu} d \mu .
\end{aligned}
$$

It remains to show that, for all $\sigma>0$,

$$
e^{-\lambda \sigma} e^{\lambda \xi} \lim _{n}\left\|\int_{\gamma_{n}^{+}-\gamma_{n}^{-}} \frac{e^{(\mu-\lambda) \xi}}{\mu-\lambda} \frac{q(\mu)}{\mu^{b}} d \mu\right\| \stackrel{\lambda \rightarrow \infty}{\longrightarrow} 0 .
$$

But, if $\lambda>\omega$,

$$
\begin{aligned}
e^{-\lambda \sigma} \| \int_{\gamma_{n}^{+}} \frac{e^{\mu \xi}}{\mu-\lambda} \frac{q(\mu)}{\mu^{b}} d \mu \mid & \leq e^{-\lambda \sigma} \int_{0}^{n} \frac{e^{\xi \operatorname{Re} \gamma^{+}(t)}}{\left|\gamma^{+}(r)-\lambda\right|} \cdot \frac{\left\|q\left(\gamma^{+}(r)\right)\right\|}{\left|\gamma^{+}(r)\right|^{b}} \cdot\left|\frac{d}{d r} \gamma^{+}(r)\right| d r \\
& \leq C_{1} C_{2} e^{-\lambda \sigma} e^{\xi \omega} \int_{0}^{n} \frac{e^{\xi r}}{\left|\gamma^{+}(r)-\lambda\right|} e^{-a b r} d r \\
& \leq C_{1} C_{2}\left(\sup _{r \geq 0} \frac{1}{\left|\gamma^{+}(r)-\lambda\right|}\right) e^{\xi \omega} e^{-\lambda \sigma} \int_{0}^{\infty} e^{(\xi-a b) r} d r \stackrel{\lambda \rightarrow \infty}{\longrightarrow} 0,
\end{aligned}
$$

where if $\lambda$ is large enough,

$$
\sup _{r \geq 0} \frac{1}{\left|\gamma^{+}(r)-\lambda\right|} \leq 1
$$


In the same way we obtain

$$
e^{-\lambda \sigma}\left\|\int_{\gamma_{n}^{-}} \frac{e^{\mu \xi}}{\mu-\lambda} \frac{q(\mu)}{\mu^{b}} d \mu\right\| \stackrel{\lambda \rightarrow \infty}{\longrightarrow} 0 .
$$

This shows

$$
q(\lambda) \sim_{\xi} \lambda^{b} \widehat{g}_{\xi}(\lambda) \quad \forall \xi \in(0, a b)
$$

Finally, if $0<b^{\prime}<b$, then $g_{b}=g_{b^{\prime}}^{\left[b-b^{\prime}\right]}$ on $\left[0, a b^{\prime}\right)$.

We have

$$
\begin{gathered}
q(\lambda) \sim_{\xi} \lambda^{b^{\prime}} \cdot\left(g_{b^{\prime}}\right)_{\xi}^{\wedge}(\lambda) \quad \forall \xi \in\left(0, a b^{\prime}\right), \\
q(\lambda) \sim_{\xi} \lambda^{b} \cdot\left(g_{b}\right)_{\xi}^{\wedge}(\lambda) \quad \forall \xi \in(0, a b),
\end{gathered}
$$

thus if $\xi \in\left(0, a b^{\prime}\right)$,

$$
\left(g_{b}\right)_{\xi}^{\wedge}(\lambda) \sim \xi \frac{1}{\lambda^{b-b^{\prime}}}\left(g_{b^{\prime}}\right) \hat{\xi}(\lambda) \sim_{\xi}\left(g_{b^{\prime}}^{\left[b-b^{\prime}\right]}\right)_{\xi}^{\wedge}(\lambda)
$$

The Phragmén Doetsch inversion formula (see, e.g., [4]) shows that $g_{b}=g_{b^{\prime}}^{\left[b-b^{\prime}\right]}$ on $\left[0, a b^{\prime}\right)$.

Theorem 3.3. Let $\alpha \in \mathbb{R}, \omega, c, a>0, F(t)=c\left(e^{a t}-1\right)$, and $M:=\{z \in \mathbb{C}|\operatorname{Re} z>\omega,| \operatorname{Im} z \mid$ $<F(\operatorname{Re} z-\omega)\}$.

Suppose $p:[0, \infty) \rightarrow \mathbb{C}$ is locally integrable with $|p(t)| \leq$ const $\cdot e^{\omega t}$ for all large $t$ and with

$$
\begin{gathered}
\liminf _{\lambda \rightarrow \infty} \frac{\ln |\hat{p}(\lambda)|}{\lambda} \geq 0, \\
\sup _{\lambda \in M}|\lambda|^{\alpha-1} \cdot|\hat{p}(\lambda)|<\infty .
\end{gathered}
$$

Furthermore, let $X$ be a complex Banach space, let $A: D(A) \rightarrow X$ be linear with $M \subset \rho(A)$, and let

$$
\sup _{\lambda \in M}|\lambda|^{\alpha} \cdot\|\hat{p}(\lambda) R(\lambda, A)\|<\infty
$$

Then, for all $m \in(-\infty, \alpha-1)$, the operator A generates a local $p^{(m-1)}$-convoluted semigroup on $[0, a(\alpha-m-1))$.

Remark 3.4. (i) Condition (3.25) can be omitted if one then assumes in addition that

$$
\limsup _{\lambda \rightarrow \infty} \frac{\ln || R(\lambda, A) \|}{\lambda} \leq 0
$$


(ii) From condition (3.25), it follows that

$$
\limsup _{\lambda \rightarrow \infty} \frac{\ln |\hat{p}(\lambda)|}{\lambda}=0,
$$

which is equivalent to $0 \in \operatorname{supp} p$, that is, $p$ does not vanish on any interval $[0, \varepsilon), \varepsilon>0$.

This can be shown by using the Phragmén Doetsch inversion formula.

Proof. We first show that $p$ is $C_{0}^{(m-1)}$ on $[0, a(\alpha-m-1))$ if $m \geq 1$, that is, there is some $g \in C([0, a(\alpha-m-1)), \mathbb{C})$ with $g(0)=0$ and $p=g^{[m-1]}$.

To this end, define $\tilde{q}: M \rightarrow \mathbb{C}$ by $\tilde{q}(\lambda)=\lambda^{\alpha-2} \hat{p}(\lambda)$.

Then we have $\sup _{\lambda \in M}|\lambda \tilde{q}(\lambda)|<\infty$. Letting $b:=\alpha-m-1$, the complex representation theorem shows that there is some $g \in C([0, a(\alpha-m-1))$, $\mathbb{C})$ with $g(0)=0$ such that

$$
\tilde{q}(\lambda) \sim \xi \lambda^{\alpha-m-1} \hat{g}_{\xi}(\lambda) \quad \forall \xi \in(0, a(\alpha-m-1)),
$$

thus

$$
\hat{p}(\lambda) \sim \xi \frac{1}{\lambda^{m-1}} \hat{g}_{\xi}(\lambda) \sim \xi\left(g^{[m-1]}\right)_{\xi}^{\wedge}(\lambda) .
$$

The Phragmén Doetsch inversion formula shows that $p^{[1]}=g^{[m]}$ on $(0, a(\alpha-m-1))$.

Next, we define the holomorphic function

$$
q: M \longrightarrow \mathscr{L}(X, \bar{D}(A)), \quad q(\lambda)=\lambda^{\alpha-2} \hat{p}(\lambda) R(\lambda, A)
$$

where $\bar{D}(A)$ is the Banach space $D(A)$ with the norm $\|x\|_{\bar{D}(A)}=\|x\|+\|A x\|$.

If $\lambda \in M$, we obtain

$$
\begin{aligned}
\|\lambda q(\lambda)\| & \leq|\lambda|^{\alpha-1}|\hat{p}(\lambda)|(\|R(\lambda, A)\|+\|\lambda R(\lambda, A)-\mathrm{id}\|) \\
& \leq|\lambda|^{\alpha-1}|\hat{p}(\lambda)|(\|R(\lambda, A)\|+|\lambda| \cdot\|R(\lambda, A)\|+1) \\
& \leq|\lambda|^{\alpha-1}|\hat{p}(\lambda)|+|\lambda|^{\alpha-1}|\hat{p}(\lambda)| \cdot(1+|\lambda|) \cdot\|R(\lambda, A)\| .
\end{aligned}
$$

From conditions (3.26) and (3.27), it follows that

$$
\sup _{\lambda \in M}\|\lambda q(\lambda)\|<\infty
$$

Letting $b:=\alpha-m-1$, the complex representation theorem yields some continuous function $H:[0, a(\alpha-m-1)) \rightarrow \mathscr{L}(X, \bar{D}(A))$ with $H(0)=0$ and with

$$
q(\lambda) \sim_{\xi} \lambda^{\alpha-m-1} \hat{H}_{\xi}(\lambda) \quad \forall \xi \in(0, a(\alpha-m-1)) .
$$

Consequently, if $\xi \in(0, a(\alpha-m-1))$ and $x \in X$,

$$
\lambda\left(p^{(m-1)}\right)_{\xi}^{\wedge}(\lambda) R(\lambda, A) x \sim \xi \lambda^{m} \hat{p}_{\xi}(\lambda) R(\lambda, A) x
$$

since $\|R(\lambda, A)\| \sim_{0} 0$ by conditions (3.25) and (3.27), and since $\left(p^{(m-1)}\right)_{\xi}^{\wedge}(\lambda) \sim_{\xi} \lambda^{m-1} \hat{p}_{\xi}(\lambda)$. 
Moreover,

$$
\lambda^{\alpha-2} \hat{p}_{\xi}(\lambda) R(\lambda, A) x \sim \xi q(\lambda) x \sim \xi \lambda^{\alpha-m-1}(H(\cdot) x)_{\xi}^{\wedge}(\lambda)
$$

Thus,

$$
\begin{aligned}
\lambda\left(p^{(m-1)}\right)_{\xi}^{\wedge}(\lambda) R(\lambda, A) x & \sim \xi \lambda^{m-\alpha+2} \lambda^{\alpha-2} \hat{p}_{\xi}(\lambda) R(\lambda, A) x \\
& \sim \xi \lambda(H(\cdot) x)_{\xi}^{\wedge}(\lambda) \\
& \sim \xi \int_{0}^{\tau} e^{-\lambda t} d_{t}(H(t) x) .
\end{aligned}
$$

Therefore, by the theory of local convoluted semigroups, the unique solution of the abstract Cauchy problem

$$
\begin{gathered}
A u_{x}(t)+p^{(m-1)}(t) x=u_{x}^{\prime}(t) \quad \forall t \in[0, a(\alpha-m-1)), \\
u_{x}(0)=0, \\
u_{x} \in C^{1}([0, a(\alpha-m-1)), X),
\end{gathered}
$$

is given by

$$
u_{x}(t)=H(t) x
$$

We now state and prove a lemma which we will need frequently in the sequel.

Lemma 3.5. Let $\tau>0, \beta>0$, let $h:[0, \infty) \rightarrow[0, \infty)$ be some function, and $\Gamma:=\{z \in \mathbb{C} \mid$ $\operatorname{Re} z>0,|\operatorname{Im} z| \leq h(\operatorname{Re} z)\}$.

Then

$$
\lim _{\substack{\operatorname{Re} \lambda \rightarrow \infty \\ \lambda \in \Gamma}} \lambda^{\beta} e^{-\lambda \tau}=0 \Longleftrightarrow \lim _{t \rightarrow \infty} e^{-(\tau / \beta) t} h(t)=0
$$

In one of these cases, even if $\beta \geq 0$,

$$
\lim _{\substack{\operatorname{Re} \lambda \rightarrow \infty \\ \lambda \in \Gamma}} \lambda^{\beta+1} \int_{\tau}^{\infty} e^{-\lambda t}\left(d \cdot t^{\beta}+g^{[\beta+1]}(t)\right) d t=0
$$

whenever $d \in \mathbb{C}$ and $g \in L_{1}^{\text {loc }}([0, \infty), \mathbb{C})$ is exponentially bounded for large arguments.

Moreover, if $\beta>0$,

$$
\limsup _{\substack{\operatorname{Re} \lambda \rightarrow \infty \\ \lambda \in \Gamma}}\left|\lambda^{\beta} e^{-\lambda \tau}\right|<\infty \Longleftrightarrow \limsup _{t \rightarrow \infty} e^{-(\tau / \beta) t} h(t)<\infty .
$$


Proof. We prove (3.41).

"If" part. If $\lambda \in \Gamma$, we obtain

$$
\begin{aligned}
\left|\lambda^{\beta} e^{-\lambda \tau}\right|^{2 / \beta} & =\left(|\operatorname{Im} \lambda|^{2}+|\operatorname{Re} \lambda|^{2}\right) e^{-(2 \tau / \beta) \operatorname{Re} \lambda} \\
& \leq\left(h(\operatorname{Re} \lambda) e^{-(\tau / \beta) \operatorname{Re} \lambda}\right)^{2}+|\operatorname{Re} \lambda|^{2} e^{-(2 \tau / \beta) \operatorname{Re} \lambda} .
\end{aligned}
$$

"Only if" part. Suppose there are some $\varepsilon>0$ and numbers $x_{n} \in \mathbb{R}$ with $x_{n} \rightarrow \infty$ and with $\left|e^{-(\tau / \beta) x_{n}} h\left(x_{n}\right)\right| \geq \varepsilon$ for all $n$. Then $z_{n}:=x_{n}+i \varepsilon e^{(\tau / \beta) x_{n}}$ is in $\Gamma$, and

$$
0=\lim _{n}\left|z_{n}^{\beta} e^{-z_{n} \tau}\right|^{2 / \beta}=\lim _{n}\left(x_{n}^{2}+\varepsilon^{2} e^{(2 \tau / \beta) x_{n}}\right) e^{-(2 \tau / \beta) x_{n}}=\varepsilon^{2}
$$

which is a contradiction. This shows (3.41).

Now let $f(t):=d t^{\beta}+g^{[\beta+1]}(t)$. Then $f^{\prime}(t)=d \beta t^{\beta-1}+g^{[\beta]}(t)$ is $L_{1}^{\text {loc }}$ and exponentially bounded for large arguments.

Partial integration shows that

$$
\lambda^{\beta+1} \int_{\tau}^{\infty} e^{-\lambda t} f(t) d t=\lambda^{\beta} e^{-\lambda \tau} f(\tau)+\lambda^{\beta} e^{-\lambda \tau} \int_{0}^{\infty} e^{-\lambda w} f^{\prime}(w+\tau) d w .
$$

Corollary 3.6. Let $\omega, a, c, v>0, \mu \geq 0, \beta \in(\mu / a, \mu / a+v], F(t)=c\left(e^{a t}-1\right)$, and $M:=$ $\{z \in \mathbb{C}|\operatorname{Re} z>\omega,| \operatorname{Im} z \mid<F(\operatorname{Re} z-\omega)\}$.

Furthermore, let $X$ be a complex Banach space, let $A: D(A) \rightarrow X$ be linear with $M \subset$ $\rho(A)$, with

$$
\limsup _{\lambda \rightarrow \infty} \frac{\ln \|R(\lambda, A)\|}{\lambda} \leq 0
$$

and with

$$
\sup _{\lambda \in M}\left\|\lambda^{\beta-\nu+1} e^{-\lambda \mu} R(\lambda, A)\right\|<\infty .
$$

Then A generates a $\nu$-times-integrated semigroup on $[0, a \beta-\mu)$.

Proof. Apply Theorem 3.3 to $m:=0, \alpha:=\beta+1$, and $p(t):=\mathbf{1}_{(\mu, \infty)}(t) \cdot(t-\mu)^{\nu-1}$, where $\mathbf{1}_{(\mu, \infty)}$ is the characteristic function on $(\mu, \infty)$. Then we have $\hat{p}(\lambda)=e^{-\lambda \mu}\left(\Gamma(\nu) / \lambda^{\nu}\right)$ if $\operatorname{Re} \lambda>0$.

Condition (3.25) is omitted, see Remark 3.4.

Condition (3.26) is equivalent to $\sup _{\lambda \in M}\left|\lambda^{\beta-\gamma} e^{-\lambda \mu}\right|<\infty$. This follows from Lemma 3.5. Here we need that $\beta \leq \mu / a+v$.

Condition (3.27) is equivalent to $\sup _{\lambda \in M}\left\|\lambda^{\beta+1-v} e^{-\lambda \mu} R(\lambda, A)\right\|<\infty$.

By Theorem 3.3 the operator $A$ generates a local $p^{[1]}$-convoluted semigroup on $[0, a \beta)$. 
If $x \in X$, let $u_{x}$ denote the (unique) solution of the abstract Cauchy problem

$$
\begin{gathered}
A u_{x}(t)+p^{[1]}(t) x=u_{x}^{\prime}(t) \quad \text { if } 0 \leq t<a \beta, \\
u_{x}(0)=0, \\
u_{x} \in C^{1}([0, a \beta), X) .
\end{gathered}
$$

The uniqueness property follows from the Ljubic uniqueness theorem.

Then $u_{x}(t)=0$ if $0 \leq t \leq \mu$, since $p^{[1]}(t)=0$ if $0 \leq t \leq \mu$. Thus if $x \in X$, then

$$
\begin{gathered}
v_{x}:[0, a \beta-\mu) \rightarrow X, \\
v_{x}(t)=u_{x}(t+\mu),
\end{gathered}
$$

is the (unique) solution of the abstract Cauchy problem

$$
\begin{gathered}
A v_{x}(t)+\frac{t^{\nu}}{v \cdot x}=v_{x}^{\prime}(t) \quad \text { if } 0 \leq t<a \beta-\mu, \\
v_{x}(0)=0 \\
v_{x} \in C^{1}([0, a \beta-\mu), X) .
\end{gathered}
$$

Corollary 3.7. Let $X$ be a complex Banach space, let $A: D(A) \rightarrow X$ be linear, $\omega, c, a>0$, $F(t)=c\left(e^{a t}-1\right)$, and $M=\{z \in \mathbb{C}|\operatorname{Re} z>\omega,| \operatorname{Im} z \mid<F(\operatorname{Re} z-\omega)\}$.

Assume that $M \subset \rho(A)$ and

$$
\sup _{\lambda \in M} \frac{\|R(\lambda, A)\|}{|\lambda|^{\gamma}}<\infty \quad \text { for some } \gamma \in[-1, \infty) .
$$

Then, for all $\varepsilon>0$, the operator A generates $a(\gamma+1+\varepsilon)$-integrated semigroup on $[0, a \varepsilon)$.

Proof. Apply Corollary 3.6 to $\mu:=0, v:=\gamma+\varepsilon+1$, and $\beta:=\varepsilon$.

Remark 3.8. Corollary 3.7 improves [7, Theorem II] if $D(A)$ is dense.

To see this we abbreviate "Cioranescu/Lumer" as "(CL)" and refer to the notation in [7].

In that theorem, put $\Phi^{(\mathrm{CL})}(r):=\ln r, \alpha^{(\mathrm{CL})}:=1 / a, \gamma^{(\mathrm{CL})}:=\gamma, K^{(\mathrm{CL})}:=h^{[\gamma+\varepsilon+1]}$, and $l^{(\mathrm{CL})}:=\gamma+\varepsilon+1$. Then $\sigma^{(\mathrm{CL})}=1$ and $\chi^{(\mathrm{CL})}=0$.

If $\omega$ is large enough, then $M \subset \Gamma_{\alpha^{(\mathrm{CL})}, \beta^{(\mathrm{CL})} \text {. }}^{(\mathrm{CL})}$

To apply [7, Theorem II] we have to make the assumptions that $\gamma>-1$, that $h^{[\gamma+\varepsilon+1]}$ is exponentially bounded, and that $\widehat{K^{(\mathrm{CL})}} \neq 0$ for all $\lambda$ with large real part. Then, by [7, Theorem II], $A$ generates a local $h^{[\gamma+\varepsilon+2]}$-convoluted semigroup on $\left[0, \tau^{(\mathrm{CL})}\right)=\left[0,\left(l^{(\mathrm{CL})}-\right.\right.$ $\left.\left.\gamma^{(\mathrm{CL})}-\sigma^{(\mathrm{CL})}\right) / \alpha^{(\mathrm{CL})}\right)=[0, a \varepsilon)$.

Example 3.9. Let $X=C([0,1], \mathbb{C})$, let $g \in X$ with $g(t)>0$ for all $t \in[0,1]$, and let $D(A)=$ $\left\{f \in C^{1}([0,1], \mathbb{C}) \mid f(0)=0\right\}$.

Then the operator $A h=-g h^{\prime}$ generates a $(1+\varepsilon)$-integrated semigroup on $[0, \infty)$ for all $\varepsilon>0$. 
If $\lambda \in \mathbb{C}$, we have

$$
R(\lambda, A) f(t)=e^{-\lambda G(t)} \int_{0}^{t} \frac{e^{\lambda G(s)} f(s)}{g(s)} d s,
$$

where $G(t)=\int_{0}^{t}(d s / g(s)) d s$.

The assertion follows from Corollary 3.7 since $\sup _{\operatorname{Re} \lambda>0}\|R(\lambda, A)\|<\infty$.

Corollary 3.10. Let $X$ be a complex Banach space, let $A: D(A) \rightarrow X$ be linear, $\omega, c, a>0$, $F(t)=c\left(e^{a t}-1\right)$, and $M=\{z \in \mathbb{C}|\operatorname{Re} z>\omega,| \operatorname{Im} z \mid<F(\operatorname{Re} z-\omega)\}$.

Assume that $M \subset \rho(A)$, that

$$
\limsup _{\lambda \rightarrow \infty} \frac{\ln || R(\lambda, A)||}{\lambda} \leq 0
$$

and that

$$
\sup _{\lambda \in M}\left\|e^{-\lambda \mu} R(\lambda, A)\right\|<\infty \quad \text { for some } \mu \geq 0 .
$$

Then for all $\varepsilon>\mu / a$, the operator A generates a $(\varepsilon+1)$-integrated semigroup on $[0, a \varepsilon-\mu)$.

Proof. Apply Corollary 3.6 to $\nu:=1+\varepsilon$ and $\beta:=\varepsilon$.

Theorem 3.11. Let $\beta \geq 0, \tau>0, g \in L_{1}([0, \tau], \mathbb{K}), c \in \mathbb{K} \backslash\{0\}$, and $f(t):=c t^{\beta}+g^{[\beta+1]}(t)$.

Furthermore, let $h:[0, \infty) \rightarrow[0, \infty)$ be some function with $h(t)=o\left(e^{(\tau / \beta) t}\right)$ as $t \rightarrow \infty$ (this condition is always fulfilled if $\beta=0$. In this case $\Gamma$ is supposed to be some right halfplane in $\mathbb{C}$ ), and let

$$
\Gamma:=\{z \in \mathbb{C}|| \operatorname{Im} z \mid \leq h(\operatorname{Re} z)\}
$$

Finally, let $X$ be some real or complex Banach space and let $A: D(A) \rightarrow X$ be some linear and closed operator which generates a local $f$-convoluted semigroup on $[0, \tau]$.

Then there is some $\omega>0$ such that $R(\lambda, A)$ exists if $\lambda \in \Gamma$ with $\operatorname{Re} \lambda>\omega$, and

$$
\sup _{\substack{\operatorname{Re} \lambda>\omega \\ \lambda \in \Gamma}} \operatorname{Re} \lambda \cdot \frac{\|R(\lambda, A)\|}{|\lambda|^{\beta}}<\infty .
$$

Proof. From Lemma 3.5, it follows that

$$
\lim _{\substack{\operatorname{Re} \lambda \rightarrow \infty \\ \lambda \in \Gamma}} \lambda^{\beta+1} \hat{f}_{\tau}(\lambda)=c \cdot \Gamma(\beta+1) .
$$

Thus, again by Lemma 3.5,

$$
\left|\lambda e^{\lambda \tau} \hat{f}_{\tau}(\lambda)\right|=\left|\lambda^{-\beta} e^{\lambda \tau}\right| \cdot\left|\lambda^{\beta+1} \hat{f}_{\tau}(\lambda)\right| \longrightarrow \infty \quad \text { if } \operatorname{Re} \lambda \longrightarrow \infty, \lambda \in \Gamma,
$$

which also holds if $\beta=0$. 
From the theory of local convoluted semigroups (see, e.g., $[8,11]$ ), it follows that there is some $\omega>0$ such that $R(\lambda, A)$ exists if $\operatorname{Re} \lambda>\omega, \lambda \in \Gamma$, and that

$$
\|R(\lambda, A)\| \leq \frac{\text { const }}{\operatorname{Re} \lambda \cdot\left|\lambda \hat{f}_{\tau}(\lambda)\right|}
$$

To be more precise, let $u_{x}$ denote the solution of the abstract Cauchy problem

$$
\begin{gathered}
A u_{x}(t)+f(t) x=u_{x}^{\prime}(t) \quad \text { if } 0 \leq t \leq \tau, \\
u_{x}(0)=0, \\
u_{x} \in C^{1}([0, \tau], X) .
\end{gathered}
$$

Let $H x:=A u_{x}(\tau)$ and $J_{\lambda}:=\lambda \hat{f}_{\tau}(\lambda) \cdot \mathrm{id}-e^{-\lambda \tau} H$. From

$$
\frac{J_{\lambda}}{\lambda \hat{f}_{\tau}(\lambda)}=\mathrm{id}-\frac{H}{\lambda e^{\lambda \tau} \hat{f}_{\tau}(\lambda)},
$$

it follows that $J_{\lambda}$ is invertible if $\lambda \in \Gamma$ and $\operatorname{Re} \lambda$ is large, and

$$
\left(\frac{J_{\lambda}}{\lambda \hat{f}_{\tau}(\lambda)}\right)^{-1}=\sum_{k=0}^{\infty}\left(\frac{H}{\lambda e^{\lambda \tau} \hat{f}_{\tau}(\lambda)}\right)^{k} .
$$

Thus, $\left\|J_{\lambda}^{-1}\right\| \leq 2 /\left|\lambda \hat{f}_{\tau}(\lambda)\right|$ if $\lambda \in \Gamma$ and $\operatorname{Re} \lambda$ is large.

From

$$
(\lambda-A) \int_{0}^{\tau} e^{-\lambda t} d u_{x}(t)=J_{\lambda} x \quad \forall x \in X, \lambda \in \mathbb{K}
$$

it follows that $R(\lambda, A)$ exists, and

$$
\|R(\lambda, A)\| \leq \frac{\text { const }}{\operatorname{Re} \lambda \cdot\left|\lambda \hat{f}_{\tau}(\lambda)\right|} .
$$

Example 3.12. Let $X=C([0,1], \mathbb{C})$ and $D(A)=\left\{f \in C^{2}([0,1], \mathbb{C}) \mid f(0)=f^{\prime}(0)=0\right\}$. Then $A h=-h^{\prime \prime}$ does not generate a local $\beta$-integrated semigroup on $[0, \tau]$, independent of $\beta \geq 0$ and $\tau>0$.

We have, if $\lambda \in \mathbb{C}$,

$$
R\left(-\lambda^{2}, A\right) f(t)= \begin{cases}\frac{1}{\lambda} \int_{0}^{t} \sinh (\lambda s) f(t-s) d s & \text { if } \lambda \neq 0 \\ \int_{0}^{t} s f(t-s) d s & \text { if } \lambda=0 .\end{cases}
$$

Let $\Gamma:=\{z \in \mathbb{C}|| \operatorname{Im} z|\leq 4 / 3 \cdot| \operatorname{Re} z \mid\}$ and consider $\mu(\alpha)=-\alpha+2 i \alpha$ if $\alpha>0$. 
Then $-\mu^{2}=3 \alpha^{2}+4 i \alpha^{2} \in \Gamma$, and

$$
\frac{\left\|R\left(-\mu^{2}, A\right)\right\|}{|\mu|^{2 \beta}} \geq \frac{\left\|R\left(-\mu^{2}, A\right)(\mathbf{1})\right\|}{|\mu|^{2 \beta}} \stackrel{\alpha \rightarrow \infty}{\longrightarrow} \infty,
$$

where $\mathbf{1}(t)=1$.

Theorem 3.13. Let $X$ be a complex Banach space and $A: D(A) \rightarrow X$ a linear operator.

Then the following assertions are equivalent.

(1) There are $\beta \geq 0$ and $\xi>0$ such that $A$ generates a $\beta$-times integrated semigroup on $[0, \xi)$.

(2) There are $c, \omega, a>0$ such that if $F(t):=c\left(e^{a t}-1\right)$ and $M:=\{z \in \mathbb{C} \mid \operatorname{Re} z>\omega$, $|\operatorname{Im} z| \leq F(\operatorname{Re} z-\omega)\}$, the following is valid:

$$
\begin{gathered}
M \subset \rho(A), \\
\sup _{\lambda \in M}\left\|\frac{e^{-\lambda \mu}}{\lambda^{\tilde{\beta}}} R(\lambda, A)\right\|<\infty \quad \text { for some } \mu, \tilde{\beta} \in \mathbb{R}, \\
\limsup _{\lambda \rightarrow \infty} \frac{\ln || R(\lambda, A) \|}{\lambda} \leq 0 .
\end{gathered}
$$

(3) There are $\omega, \delta>0$ such that if $h(t):=e^{\delta t}$ and $\Gamma:=\{z \in \mathbb{C}|\operatorname{Re} z>\omega,| \operatorname{Im} z \mid \leq h(\operatorname{Re} z)\}$, the following is valid:

$$
\begin{gathered}
\Gamma \subset \rho(A), \\
\sup _{\lambda \in \Gamma}\left\|\frac{R(\lambda, A)}{\lambda^{\bar{\beta}}}\right\|<\infty \quad \text { for some } \bar{\beta} \in \mathbb{R} .
\end{gathered}
$$

Proof. $(1) \Rightarrow(3)$. Choose $\delta \in(0, \xi / \beta)$, where $\xi / \beta:=\infty$ if $\beta=0$. Apply Theorem 3.11 to $g:=0, c:=1, \bar{\beta}:=\beta$ and choose $\tau \in(\beta \delta, \xi)$. Then $h(t)=e^{\delta t}$ fulfills $h(t)=o\left(e^{(\tau / \beta) t}\right)$, thus, by Theorem 3.11, there is some $\omega>0$ such that $R(\lambda, A)$ exists if $\operatorname{Re} \lambda>\omega, \lambda \in \Gamma$, and

$$
\sup _{\substack{\operatorname{Re} \lambda>\omega \\ \lambda \in \Gamma}} \frac{\|R(\lambda, A)\|}{|\lambda| \bar{\beta}}<\infty .
$$

Here we needed the uniqueness property on $[0, \tau]$, see [10, Theorem 3.5.1].

(3) $\Rightarrow(2)$. Choose $a:=\delta, c:=1$, and numbers $\mu \geq 0, \tilde{\beta} \in \mathbb{R}$ such that $\delta \cdot(\bar{\beta}-\tilde{\beta}) \leq \mu$. For example, take $\mu:=0$ and $\tilde{\beta}:=\bar{\beta}$.

Then $M \subset \Gamma$, and if $\lambda \in M$,

$$
\left\|\frac{e^{-\lambda \mu}}{\lambda^{\tilde{\beta}}} R(\lambda, A)\right\|=\left|\lambda^{\bar{\beta}-\tilde{\beta}} e^{-\lambda \mu}\right| \cdot\left\|\frac{R(\lambda, A)}{\lambda^{\bar{\beta}}}\right\| .
$$


If $\bar{\beta}-\tilde{\beta}>0$, then

$$
\limsup _{\substack{\operatorname{Re} \lambda \rightarrow \infty \\ \lambda \in M}}\left|\lambda^{\bar{\beta}-\tilde{\beta}} e^{-\lambda \mu}\right|<\infty
$$

by Lemma 3.5, since

$$
\limsup _{t \rightarrow \infty} e^{-(\mu /(\bar{\beta}-\tilde{\beta})) t} h(t)<\infty .
$$

(2) $\Rightarrow(1)$. First, let $\tilde{\beta} \geq-\mu / a-1$. Choose $\nu>\mu / a+1+\tilde{\beta}$ and $\hat{\beta}:=v-\tilde{\beta}-1$. Then $\hat{\beta} \in$ $(\mu / a, \mu / a+\nu]$ and

$$
\left\|\lambda^{\hat{\beta}-\nu+1} e^{-\lambda \mu} R(\lambda, A)\right\| \leq\left\|\frac{e^{-\lambda \mu}}{\lambda^{\tilde{\beta}}} R(\lambda, A)\right\| \quad \forall \lambda \in M, \operatorname{Re} \lambda>\omega .
$$

Corollary 3.6 shows that $A$ generates a local $\nu$-times integrated semigroup on $[0, a(\nu-$ $1-\tilde{\beta})-\mu)$.

Second, assume $\tilde{\beta} \leq-\mu / a-1$. Let $\nu>0$ and $\hat{\beta}=\mu / a+\nu$. Then $\hat{\beta} \in(\mu / a, \mu / a+\nu]$ and, if $\omega>1$,

$$
\left\|\lambda^{\hat{\beta}-\nu+1} e^{-\lambda \mu} R(\lambda, A)\right\| \leq\left\|\frac{e^{-\lambda \mu}}{\lambda^{\tilde{\beta}}} R(\lambda, A)\right\| \quad \forall \lambda \in M, \operatorname{Re} \lambda>\omega,
$$

since $\tilde{\beta}+\hat{\beta}-\nu+1 \leq 0$.

Corollary 3.6 shows that $A$ generates a local $\nu$-times integrated semigroup on $[0, a v)$.

\section{The theorem of Prüss and its application}

Next we generalize a result from Prüss [12, Theorem 0.4] to the local case. In its original form, the function $q$ has to be defined on some right half-plane and then is the Laplace transform of some continuous and exponentially bounded function.

This will lead to a new Hille-Yosida-type theorem for integrated semigroups.

Theorem 4.1. Let $X$ be a complex Banach space, $c, a, \omega_{1}>0, F(t):=c\left(e^{a t}-1\right)$, and $M:=$ $\left\{z \in \mathbb{C}\left|\operatorname{Re} z>\omega_{1},\right| \operatorname{Im} z \mid<F\left(\operatorname{Re} z-\omega_{1}\right)\right\}$.

Furthermore, let $q: M \rightarrow X$ be a holomorphic function satisfying

$$
\sup _{\lambda \in M}\|\lambda q(\lambda)\|<\infty, \quad \sup _{\lambda \in M}\left\|\lambda^{2} q^{\prime}(\lambda)\right\|<\infty .
$$

Then there is some $H \in C((0, a), X)$ such that $t \mapsto(t-a) H(t)$ is bounded on $(0, a)$ and with

$$
q(\lambda) \sim_{\xi} \hat{H}_{\xi}(\lambda) \quad \forall \xi \in(0, a) .
$$


Proof. From the complex representation theorem it follows that there are functions $g, h \in$ $C([0, a), X)$ such that $g(t) / t$ and $h(t) / t$ are in $L_{\infty}^{\text {loc }}([0, a), X)$ and with

$$
\begin{gathered}
q(\lambda)=\lambda \int_{0}^{\xi} e^{-\lambda t} g(t) d t+\varepsilon_{\xi}(\lambda), \\
\lambda q^{\prime}(\lambda)=\lambda \int_{0}^{\xi} e^{-\lambda t} h(t) d t+\psi \xi(\lambda),
\end{gathered}
$$

for all $\xi \in(0, a)$, where $\varepsilon \xi$ and $\psi \xi$ are functions satisfying

$$
\limsup _{\lambda \rightarrow \infty} \frac{\ln || \varepsilon_{\xi}(\lambda) \|}{\lambda} \leq-\xi, \quad \limsup _{\lambda \rightarrow \infty} \frac{\ln \|\psi \xi(\lambda)\|}{\lambda} \leq-\xi .
$$

Thus, if $\eta>\omega>\omega_{1}$, then

$$
\begin{aligned}
\int_{\omega}^{\eta} q^{\prime}(\lambda) d \lambda & =\int_{\omega}^{\eta} \int_{0}^{\xi} e^{-\lambda t} h(t) d t d \lambda+\int_{\omega}^{\eta} \frac{\psi \xi(\lambda)}{\lambda} d \lambda \\
& =\int_{0}^{\xi} \frac{e^{-\omega t}-e^{-\eta t}}{t} h(t) d t+\int_{\omega}^{\eta} \frac{\psi \xi(\lambda)}{\lambda} d \lambda \\
& \stackrel{\eta \rightarrow \infty}{\longrightarrow} \int_{0}^{\xi} \frac{e^{-\omega t} h(t)}{t} d t+\int_{\omega}^{\infty} \frac{\psi \xi(\lambda)}{\lambda} d \lambda
\end{aligned}
$$

Thus, $y:=\lim _{\eta \rightarrow \infty} q(\eta)$ exists, and

$$
y-\omega \int_{0}^{\xi} e^{-\omega t} g(t) d t-\varepsilon \xi(\omega)=\int_{0}^{\xi} \frac{e^{-\omega t} h(t)}{t} d t+\int_{\omega}^{\infty} \frac{\psi \xi(\lambda)}{\lambda} d \lambda
$$

for all $\omega>\omega_{1}$ and all $\xi \in(0, a)$.

It is not hard to see that for all $\delta>0$ we have

$$
e^{-\omega \delta} e^{\omega \xi}\left\|\int_{\omega}^{\infty} \frac{\psi \xi(\lambda)}{\lambda} d \lambda\right\| \stackrel{\omega \rightarrow \infty}{\longrightarrow} 0,
$$

that is,

$$
\limsup _{\omega \rightarrow \infty} \frac{\ln \left\|\int_{\omega}^{\infty}(\psi \xi(\lambda) / \lambda) d \lambda\right\|}{\lambda} \leq-\xi
$$

Thus

$$
\int_{0}^{\xi} e^{-\omega t}(y-g(t)) d t \sim \xi \int_{0}^{\xi} \frac{e^{-\omega t}}{\omega} \cdot \frac{h(t)}{t} d t \sim \xi \int_{0}^{\xi} e^{-\omega t} H^{[1]}(t) d t,
$$

where $H(t):=h(t) / t$.

Consequently, $y-g(t)=H^{[1]}(t)$ for all $0 \leq t<a$. This shows that $y=0$ and

$$
g(t)=-\int_{0}^{t} \frac{h(t)}{t} d t \quad \forall 0 \leq t<a .
$$


Thus

$$
q(\lambda) \sim_{\xi} \int_{0}^{\xi} e^{-\lambda t} H(t) d t
$$

Theorem 4.2. Let $c, a, \omega_{1}>0, F(t):=c\left(e^{a t}-1\right), M:=\left\{z \in \mathbb{C}\left|\operatorname{Re} z>\omega_{1},\right| \operatorname{Im} z \mid<F(\operatorname{Re} z\right.$ $\left.\left.-\omega_{1}\right)\right\}$, and let $p:(0, \infty) \rightarrow \mathbb{C}$ be some continuous function satisfying $|p(t)| \leq$ const $\cdot e^{\omega_{1} t}$ for all $t>0$ and

$$
\begin{gathered}
\liminf _{\lambda \rightarrow \infty} \frac{\ln |\hat{p}(\lambda)|}{\lambda} \geq 0, \\
\sup _{\lambda \in M}|\lambda \hat{p}(\lambda)|<\infty, \\
\sup _{\lambda \in M}\left|\lambda^{2} \frac{d}{d \lambda} \hat{p}(\lambda)\right|<\infty .
\end{gathered}
$$

Let $X$ be a complex Banach space, $A: D(A) \rightarrow X$ linear with $M \subset \rho(A)$,

$$
\begin{gathered}
\sup _{\lambda \in M}\left|\lambda^{3} \hat{p}(\lambda)\right| \cdot\left\|R(\lambda, A)^{2}\right\|<\infty, \\
\sup _{\lambda \in M}\left|\lambda^{2} \hat{p}(\lambda)\right| \cdot\|R(\lambda, A)\|<\infty, \\
\sup _{\lambda \in M}\left|\lambda^{3} \frac{d}{d \lambda} \hat{p}(\lambda)\right| \cdot\|R(\lambda, A)\|<\infty .
\end{gathered}
$$

Then the following assertions hold:

(1) A generates a local $p^{[1]}$-convoluted semigroup on $[0, a)$;

(2) the abstract Cauchy problem

$$
\begin{gathered}
A v(t)+p(t) x=v^{\prime}(t) \quad \text { if } 0<t<a, \\
v(0)=0, \\
v \in C^{1}((0, a), X) \cap C([0, a), X),
\end{gathered}
$$

has a unique solution $v_{x}$ for all $x \in X$;

(3) if $p$ can be continuously extended in 0 , then $A_{\overline{D(A)}}$ generates a local p-convoluted semigroup on $[0, a)$.

Remark 4.3. Condition (4.12) can be omitted if one then assumes in addition that

$$
\limsup _{\lambda \rightarrow \infty} \frac{\ln || R(\lambda, A) \|}{\lambda} \leq 0
$$

Proof. Consider the holomorphic function

$$
q: M \longrightarrow \mathscr{L}(X, \bar{D}(A)), \quad q(\lambda)=\hat{p}(\lambda) R(\lambda, A)
$$


Then $\lambda^{2} q^{\prime}(\lambda)=-\lambda^{2} \hat{p}(\lambda) R(\lambda, A)^{2}+\lambda^{2}(d \hat{p}(\lambda) / d \lambda) R(\lambda, A)$, thus

$$
\begin{aligned}
\left\|\lambda^{2} q^{\prime}(\lambda)\right\| \leq & \left\|\lambda^{2} \hat{p}(\lambda) R(\lambda, A)^{2}\right\|+\left\|\lambda^{2} \frac{d \hat{p}(\lambda)}{d \lambda} R(\lambda, A)\right\| \\
& +\left\|(\lambda R(\lambda, A)-\mathrm{id})\left(\lambda^{2} \hat{p}(\lambda) R(\lambda, A)-\lambda^{2} \frac{d \hat{p}(\lambda)}{d \lambda} \cdot \mathrm{id}\right)\right\| \\
\leq & \left\|\lambda^{2} \hat{p}(\lambda) R(\lambda, A)^{2}\right\|+\left\|\lambda^{2} \frac{d \hat{p}(\lambda)}{d \lambda} R(\lambda, A)\right\|+\left\|\lambda^{3} \hat{p}(\lambda) R(\lambda, A)^{2}\right\| \\
& +\left\|\lambda^{3} \frac{d \hat{p}(\lambda)}{d \lambda} R(\lambda, A)\right\|+\left\|\lambda^{2} \hat{p}(\lambda) R(\lambda, A)\right\|+\left|\lambda^{2} \frac{d \hat{p}(\lambda)}{d \lambda}\right| .
\end{aligned}
$$

It follows from the assumptions that

$$
\sup _{\lambda \in M}\left\|\lambda^{2} q^{\prime}(\lambda)\right\|<\infty
$$

Moreover,

$$
\|\lambda q(\lambda)\| \leq\|\lambda \hat{p}(\lambda) R(\lambda, A)\|+\|\lambda \hat{p}(\lambda)(\lambda R(\lambda, A)-\mathrm{id})\|
$$

thus

$$
\sup _{\lambda \in M}\|\lambda q(\lambda)\|<\infty
$$

From Theorem 4.1 it follows that there is some $H \in C((0, a), \mathscr{L}(X, \bar{D}(A)))$ such that $t \mapsto$ $(t-a) H(t)$ is bounded on $(0, a)$ and with

$$
\hat{p}(\lambda) R(\lambda, A) \sim \xi \int_{0}^{\xi} e^{-\lambda t} H(t) d t \quad \forall \xi \in(0, a) .
$$

From conditions (4.12) and (4.16), it follows that $\limsup _{\lambda \rightarrow \infty}(\ln \|R(\lambda, A)\| / \lambda) \leq 0$, thus

$$
\begin{aligned}
\lambda\left(p^{[1]}\right)_{\xi}^{\wedge}(\lambda) R(\lambda, A) x & \sim \xi \hat{p} \xi(\lambda) R(\lambda, A) x \\
& \sim \xi \int_{0}^{\xi} e^{-\lambda t} H(t) x d t \\
& \sim \xi \int_{0}^{\xi} e^{-\lambda t} d_{t}\left(H^{[1]}(t) x\right) .
\end{aligned}
$$

This shows (1). To be precise, the solution of the abstract Cauchy problem

$$
\begin{gathered}
A u_{x}(t)+p^{[1]}(t) x=u_{x}^{\prime}(t), \quad 0 \leq t<a, \\
u_{x}(0)=0, \\
u_{x} \in C^{1}([0, a), X),
\end{gathered}
$$

is $u_{x}(t)=\int_{0}^{t} H(s) x d s$. 
Further on, we define $v_{x}:[0, a) \rightarrow X$ by

$$
v_{x}(t)= \begin{cases}0 & \text { if } t=0, \\ H(t) x & \text { if } 0<t<a .\end{cases}
$$

Since $A v_{x}^{[1]}=(A H(\cdot) x)^{[1]}$ is in $C^{1}((0, a), X)$, we have $v_{x} \in C^{1}((0, a), X)$, and since $A$ is closed, we obtain

$$
A v_{x}(t)+p(t) x=v_{x}^{\prime}(t) \quad \text { if } 0<t<a
$$

This shows (2).

Finally if $x \in D(A)$, then

$$
\begin{aligned}
& A_{\overline{D(A)}} v_{x}(t)+p(t) x=v_{x}^{\prime}(t) \quad \text { if } 0 \leq t<a \text {, } \\
& v_{x}(0)=0 \text {, } \\
& v_{x} \in C^{1}([0, a), \overline{D(A)}) \text {. }
\end{aligned}
$$

The result follows if we can show that

$$
\sup _{0 \leq t \leq \xi} \sup _{\substack{y \in D(A) \\\|y\| \leq 1}}\left\|v_{y}^{\prime}(t)\right\|_{X}<\infty \quad \forall \xi \in(0, a)
$$

If $0<t \leq \xi$ and $y \in D(A)$ with $\|y\| \leq 1$, we obtain

$$
\begin{aligned}
\left\|v_{y}^{\prime}(t)\right\| & \leq\left\|A v_{y}(t)+p(t) y\right\|=\|A H(t) y+p(t) y\| \\
& \leq\|A H(t)\|_{\mathscr{L}(X)}+\|p\|_{L_{\infty}[0, \xi] .}
\end{aligned}
$$

Corollary 4.4. Let $c, a, \omega_{1}>0, F(t):=c\left(e^{a t}-1\right)$, and $M:=\left\{z \in \mathbb{C}\left|\operatorname{Re} z>\omega_{1},\right| \operatorname{Im} z \mid<\right.$ $\left.F\left(\operatorname{Re} z-\omega_{1}\right)\right\}$.

Suppose $X$ is a complex Banach space, $A: D(A) \rightarrow X$ is linear with $M \subset \rho(A)$, and that there is some $\gamma \geq 0$ with

$$
\begin{aligned}
& \sup _{\lambda \in M} \frac{\left\|R(\lambda, A)^{2}\right\|}{|\lambda|^{\gamma-2}}<\infty, \\
& \sup _{\lambda \in M} \frac{\|R(\lambda, A)\|}{|\lambda|^{\gamma-1}}<\infty .
\end{aligned}
$$

Then the following assertions hold:

(1) A generates a $(\gamma+1)$-integrated semigroup on $[0, a)$; 
(2) the abstract Cauchy problem

$$
\begin{gathered}
A v(t)+t^{\gamma} \cdot x=v^{\prime}(t) \quad \text { if } 0<t<a, \\
v(0)=0, \\
v \in C^{1}((0, a), X) \cap C([0, a), X),
\end{gathered}
$$

has a unique solution $v_{x}$ for all $x \in X$;

(3) $A_{\overline{D(A)}}$ generates a $\gamma$-integrated semigroup on $[0, a)$.

Proof. Let $p(t)=t^{\gamma}$ and apply Theorem 4.2.

\section{References}

[1] B. Bäumer, A vector-valued operational calculus and abstract Cauchy problems, Ph.D. dissertation, Louisiana State University, Louisiana, 1997, http://math.lsu.edu/ baeumer.

[2] - Approximate solutions to the abstract Cauchy problem, Evolution Equations and Their Applications in Physical and Life Sciences (Bad Herrenalb, 1998), Lecture Notes in Pure and Appl. Math., vol. 215, Dekker, New York, 2001, pp. 33-41.

[3] B. Bäumer, G. Lumer, and F. Neubrander, Convolution kernels and generalized functions, Generalized Functions, Operator Theory, and Dynamical Systems (Brussels, 1997), Chapman \& Hall/CRC Res. Notes Math., vol. 399, Chapman \& Hall/CRC, Florida, 1999, pp. 68-78.

[4] B. Bäumer and F. Neubrander, Laplace transform methods for evolution equations, Confer. Sem. Mat. Univ. Bari (1994), no. 258-260, 27-60.

[5] I. Cioranescu and G. Lumer, Problèmes d'évolution régularisés par un noyau général $K(t)$. Formule de Duhamel, prolongements, théorèmes de génération [Evolution problems regularized via a general kernel $K(t)$. Duhamel formula, extensions, generation results], C. R. Acad. Sci. Paris Sér. I Math. 319 (1994), no. 12, 1273-1278 (French).

[6] Regularization of evolution equations via kernels $K(t)$, $K$-evolution operators and convoluted semigroups, generation theorems, LSU Seminar Notes in Functional Analysis and PDEs 1993/1994, Louisiana State University, Louisiana, 1994, pp. 45-52.

[7] - On $K(t)$-convoluted semigroups, Recent Developments in Evolution Equations (Glasgow, 1994), Pitman Res. Notes Math. Ser., vol. 324, Longman Sci. Tech., Harlow, 1995, pp. 86-93.

[8] V. Keyantuo, C. Müller, and P. Vieten, The Hille-Yosida theorem for local convoluted semigroups, Proc. Edinb. Math. Soc. (2) 46 (2003), no. 2, 395-413.

[9] G. Lumer and F. Neubrander, The asymptotic Laplace transform: new results and relation to Komatsu's Laplace transform of hyperfunctions, Partial Differential Equations on Multistructures (Luminy, 1999), Lecture Notes in Pure and Appl. Math., vol. 219, Dekker, New York, 2001, pp. 147-162.

[10] C. Müller, Local Cauchy Problems and the Finite Laplace Transform, Shaker Verlag, Aachen, 2000 (German).

[11] Approximation of local convoluted semigroups, J. Math. Anal. Appl. 269 (2002), no. 2, 401-420.

[12] J. Prüss, Evolutionary Integral Equations and Applications, Monographs in Mathematics, vol. 87, Birkhäuser Verlag, Basel, 1993.

Claus Müller: Fachbereich Mathematik, Universitaet Kaiserslautern, Erwin Schroedinger Strasse, 67655 Kaiserslautern, Germany

E-mail address: claus_mueller@mathematik.uni-kl.de 


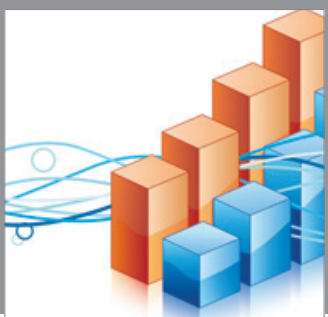

Advances in

Operations Research

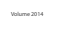

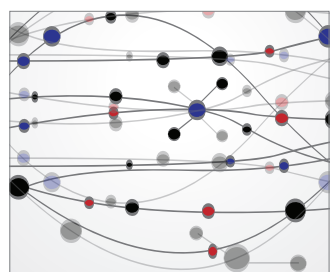

\section{The Scientific} World Journal
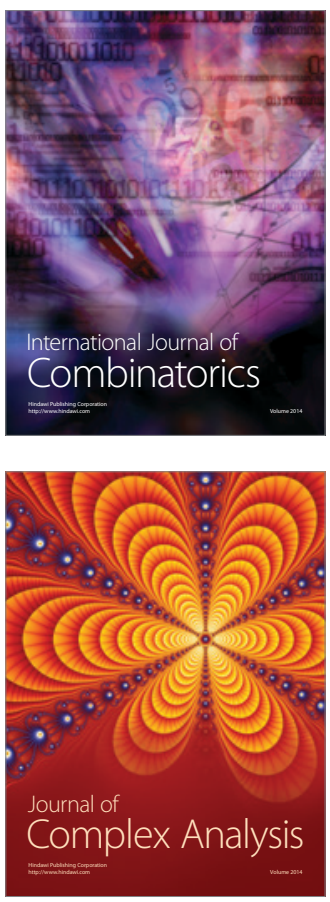

International Journal of

Mathematics and

Mathematical

Sciences
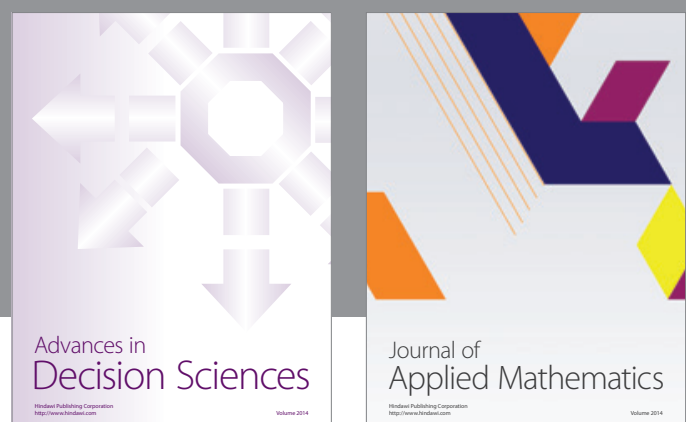

Journal of

Applied Mathematics
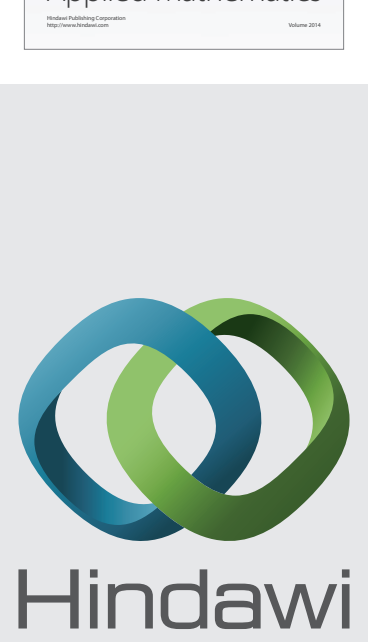

Submit your manuscripts at http://www.hindawi.com
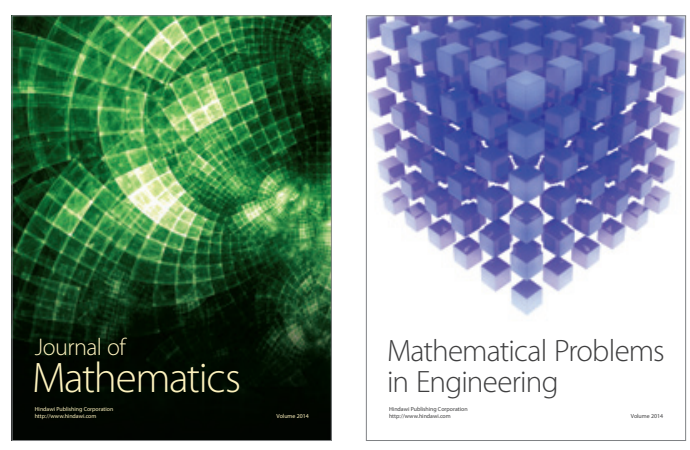

Mathematical Problems in Engineering
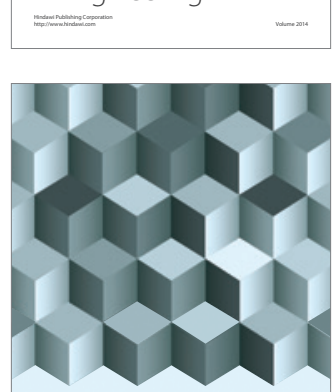

Journal of

Function Spaces
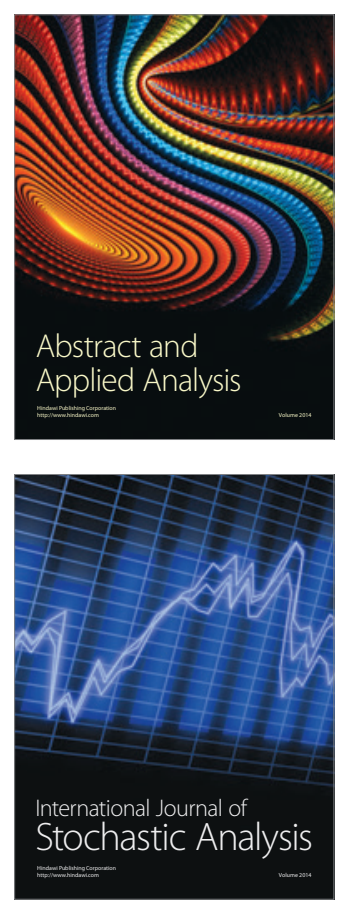

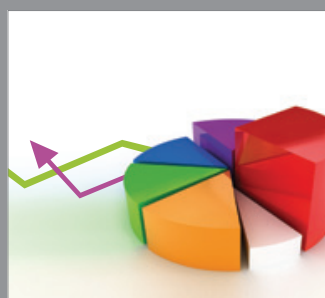

ournal of

Probability and Statistics

Promensencen
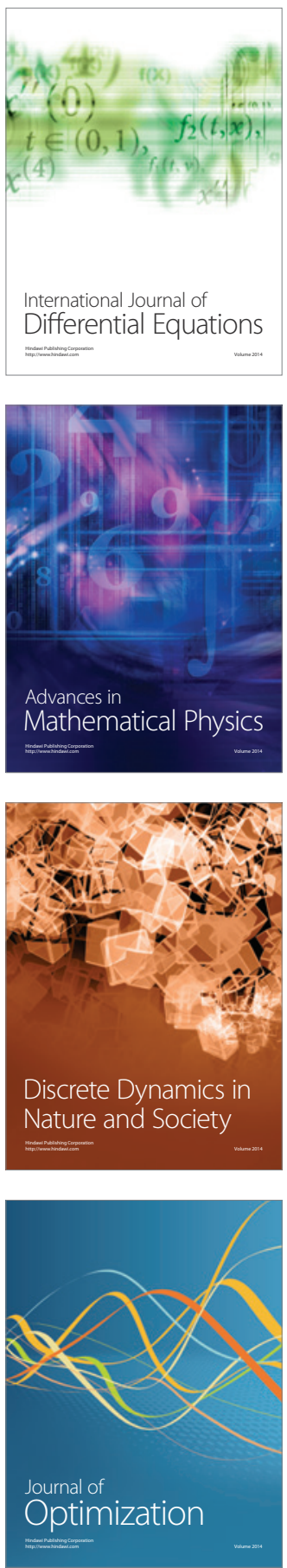